Bundesgesundheitsbl 2010 · 53:754-756

DOI 10.1007/s00103-010-1106-z

Online publiziert: 30. Juni 2010

(c) Springer-Verlag 2010

\section{Die Kategorien in der Richtlinie für Krankenhaushygiene und Infektionsprävention - Aktualisierung der Definitionen}

\author{
Mitteilung der Kommission \\ für Krankenhaushygiene und \\ Infektionsprävention
}

Im Jahr 1999 sin d er stmals Em pfehlungen der Kommission für Krankenhaushygiene un d Infektionsprävention er schienen, deren einzelne Empfehlungen mit ein er Evidenzkategorie ( $\mathrm{b}$ is IV) belegt waren [1].

Bis dahin wurden zur Verdeutlichung und Differenzierung des Em pfehlungsgrades (der V erbindlichkeit o der Notwendigkeit von einzelnen Empfehlungen) modale Hilfsverben wie ", soll“, , sollte“ oder „muss“ verwendet. Um Anwendern in Klinik und Praxis die wissenschaftliche Grundlage von Empfehlungen zu verdeutlichen und zudem Entscheidungsspielräume aufzuzeigen, entschied sich die Kom- mission für Krankenhaushygiene und Infektionsprävention, Evidenzgrade für die einzelnen Empfehlungen, basierend auf wissenschaftlichen Studien oder theoretischer Begründung, anzugeben.

Die Kriterien wurden in Anlehnung an die von den Centers for Disease Control and Prevention (CDC, Atlanta) verwendeten Kriterien entwickelt und vom Robert Koch-Institut veröffentlicht. In Ergänzung zu den d amals von den CD C verwendeten Kategorien wurde eine Kategorie IV eingeführt, um solche Anforderungen zu kennzeichnen, die g esetzliche Bestimmungen zur G rundlage haben (• Tab. 1).

\section{Tab. 1 Kategorien in der Richtlinie für Krankenhaushygiene und Infektionsprävention} (1999)

Kategorie IA: Nachdrückliche Empfehlung für alle Krankenhäuser

Die Empfehlungen basieren auf gut konzipierten experimentellen oder epidemiologischen Studien.

Kategorie IB: Nachdrückliche Empfehlung für alle Krankenhäuser

Die Empfehlungen werden von Experten und aufgrund eines Konsens-Beschlusses der Krankenhaushygiene-Kommission am Robert Koch-Institut als effektiv angesehen und basieren auf gut begründeten Hinweisen für deren Wirksamkeit. Eine Einteilung der entsprechenden Empfehlung in die Kategorie IB kann auch dann erfolgen, wenn wissenschaftliche Studien möglicherweise hierzu nicht durchgeführt wurden.

Kategorie II: Empfehlungen zur Einführung/Umsetzung in vielen Kliniken

Die Empfehlungen basieren teils auf hinweisenden klinischen oder epidemiologischen Studien, teils auf nachvollziehbaren theoretischen Begründungen oder Studien, die in einigen, aber nicht allen Kliniken anzuwenden sind.

Kategorie III: keine Empfehlung oder ungelöste Fragen

Maßnahmen, über deren Wirksamkeit nur unzureichende Hinweise vorliegen oder bislang kein Konsens besteht.

Kategorie IV: Rechtliche Vorgaben

Anforderungen, Maßnahmen und Verfahrensweisen in Krankenhäusern und anderen medizi-

nischen Einrichtungen, die aufgrund gesetzlicher Bestimmungen, durch autonomes Recht oder Verwaltungsvorschriften zu beachten sind.
Einhergehend mit der Eingruppierung in eine Evidenzkategorie wurde, wann immer möglich, die zu dies er Eingruppierung führende Literatur zitiert, um dem Anwender die Möglichkeit zu geben, die Übertragbarkeit der S tudienergebnisse auf die eigenen Patienten zu überprüfen.

Von 1999 bis 2007 sind 13 verschiedene Empfehlungen, in den en die Evidenzkategorien verwendet wurden, veröffentlicht worden $[2,3,4,5,6,7,8,9,10,11,12,131$ $4,15]$.

Für 839 Einzelempfehlungen wur de dabei eine Kategorie vergeben, wobei zirka 70\% der Empfehlungen der Evidenzkategorie IB entsprachen. Die anderen Kategorien wurden mit jeweils zirka $10 \%$ nahezu gleich häufig vergeben. Allerdings gibt es große Unterschiede zwischen einzelnen Bereichen. Während in klinischen Bereichen, zum Beispiel für die Empfehlungen zur Prävention nosokomialer Pneumonien, ein Drittel der Empfehlungen der höchsten wissenschaftlichen Evidenzkategorie IA entsprachen, wurde diese Kategorie für die Empfehlungen zur Aufbereitung von Medizinprodukten nicht einmal vergeben. Dies spiegelt jedoch auch die Realität und Ethik der medizinisch-wissenschaftlichen Forschung wieder. Während b estimmte B ehandlungsmethoden durchaus im $\mathrm{R}$ ahmen klinischer S tudien v erglichen w erden können, verbietet es die Ethik, wissentlich suboptimal aufbereitete Medizinprodukte am Menschen zu verwenden, sodass hier nicht klinische Studien, sondern Überle- 
gungen zur besten technischen Praxis im Vordergrund stehen.

Die Evidenz-Kategorisierung hat sich in den zur ückliegenden Jahren bewährt. Sie stellen in der täglichen Praxis der Hygiene eine starke Argumentationshilfe bei der Entscheidung für oder gegen die Einführung b estimmter P räventionsmaßnahmen dar.

Dennoch zeigte die A useinandersetzung mit den D efinitionen der Kategorien, $\mathrm{d}$ ass b estimmte F ormulierungen nicht $\mathrm{m}$ ehr der A rbeitsmethodik der Kommission für K rankenhaushygiene und Infektionsprävention bei der Erstellung der Empfehlungen entsprechen und fortentwickelt werden sollten. Hatte man sich 1999 noch entschlossen, den Gültigkeitsbereich für einzelne Empfehlungen in das System der Kategorien zu integrieren („für alle Krankenhäuser“ in Kategorie IA und IB; „Einführung/Umsetzung in vielen Kliniken“ in Kategorie II), so zeigt sich inzwischen, dass diese Einschränkungen nicht dem Inhalt der Empfehlungen entsprechen. Zudem zeigte sich, dass gerade die Vergabe der Kategorie III zu Missverständnissen führte. So war nicht immer klar, ob von der D urchführung ei ner Maßnahme mit Kategorie III abgeraten wurde oder ob die Evidenz nicht ausreichte, zur Effizienz der Maßnahme Stellung zu nehmen.

Dies hat die Kommission für Krankenhaushygiene und Infektionsprävention zum A nlass genommen, die Evidenzkategorien zu überarbeiten und zu konkretisieren, wo Bedarf bestand.

Hierbei wurden die folgenden Bedingungen zugrunde gelegt:

- Um die Kontinuität der Richtlinie zu wahren, sollte die vorhandene Kategorisierung überarbeitet und so weit als möglich beibehalten werden.

- Die örtlichen Einschränkungen „alle

Krankenhäuser, viele Kliniken“ sollen als Kriterium für die Kategorisierung ersatzlos entfallen.

- Die Abstufung der Kategorien erfolgt weiterhin nach Verbindlichkeit/ Nachdrücklichkeit der Empfehlung (Empfehlungsgrad), wobei die wissenschaftliche Evidenz zur entsprechenden Eingruppierung führt.

Tab. 2 Kategorien in der Richtlinie für Krankenhaushygiene und Infektionsprävention (2010)

Kategorie IA:

Diese Empfehlung basiert auf gut konzipierten systematischen Reviews oder einzelnen hochwertigen randomisierten kontrollierten Studien.

Kategorie IB:

Diese Empfehlung basiert auf klinischen oder hochwertigen epidemiologischen Studien und strengen, plausiblen und nachvollziehbaren theoretischen Ableitungen.

Kategorie II:

Diese Empfehlung basiert auf hinweisenden Studien/Untersuchungen und strengen, plausiblen und nachvollziehbaren theoretischen Ableitungen.

Kategorie III:

Maßnahmen, über deren Wirksamkeit nur unzureichende oder widersprüchliche Hinweise vorliegen, deshalb ist eine Empfehlung nicht möglich.

Kategorie IV:

Anforderungen, Maßnahmen und Verfahrensweisen, die durch allgemein geltende Rechtsvorschriften zu beachten sind.

Basierend auf diesen Vorgaben, wurden die in - Tab. 2 dargestellten neuen Definitionen für die Kategorien erarbeitet. Je höher die w issenschaftliche B eweiskraft der vorhandenen Studien ist, desto nachdrücklicher wird die Umsetzung der Maßnahme em pfohlen, $\mathrm{d}$ a es sic $\mathrm{h}$ um den derzeitigen Stand wissenschaftlicher Kenntnis und Erfahrung handelt. Mit den römischen Ziffern der Kategorie wird dem Grad der Empfehlung Ausdruck verliehen. Mit dem B uchstaben, der der Kategorie folgt, wird das Niveau der wissenschaftlichen Evidenz beschrieben. Die herangezogenen Studien sollen dabei im Hinblick auf den infektionspräventiven Wert jeweils den Endpunkt Infektion o der Tod des P atienten haben. Das Evidenzniveau A kennzeichnet Maßnahmen, deren Wirksamkeit in systematischen Reviews gut konzipierter Studien oder einzelnen hochwertigen randomisierten klinischen Studien nachgewiesen wurde, während für Maßnahmen, deren Wirksamkeit in weniger hochwertigen/aussagekräftigen klinischen Studien oder aber guten epidemiologischen Studien (zum B eispiel hochwertige Kohortenstudien) oder deren systematischen Reviews gezeigt wurden, das Evidenzniveau $B$ erhalten.

Die Kommission hat sich entschieden, sowohl bei Vorliegen systematischer Reviews gut konzipierter Studien oder randomisierter klinischer Studien als auch bei gleichzeitigem Vorliegen klinischer oder ep idemiologischer S tudien und strenger, das heißt plausibler und nachvollziehbarer theoretischer A bleitung, den höchsten Empfehlungsgrad I zu vergeben.

Die umfassendste Änderung ergibt sich für die Kategorie II, die bisher im Wesentlichen über eine Einschränkung des Gültigkeitsbereiches definiert war, die jetzt entfällt. Einschränkungen in der Zielpopulation der Empfehlung werden künftig im Text deutlich gemacht.

Liegen $\mathrm{n}$ ur hin weisende U ntersuchungen oder Studien vor, so sind auch hier strenge (plausible und nachvollziehbare) theoretische Ableitungen erforderlich, um ein e Maßnahme zu em pfehlen und dies mit Kategorie II zu bewerten.

Die plausiblen und nachvollziehbaren Ableitungen können a uf der B asis von theoretischen Überlegungen oder auf der Basis von Analogieschlüssen beziehungsweise Ableitungen a us exp erimenteller Grundlagenforschung (zum B eispiel Laborexperimente oder Tierversuche) resultieren.

Die Literatur, die zur Vergabe der Kategorien führt, wird immer als entsprechende Referenz angefügt.

Der Text der Kategorie III wurde umformuliert, um $\mathrm{zu} v$ erdeutlichen, $\mathrm{d}$ ass hier eine Empfehlung nicht möglich ist, weder für n och g egen die U msetzung der Maßnahme. Es handelt sich jedoch oft um kontrovers diskutierte Maßnahmen, für die nicht selten eine Reihe von Studien mit widersprüchlichen Ergebnissen vorliegt. Daher wird eine Stellungnahme hierzu in den Empfehlungen für erforderlich gehalten. Anhand der zitierten Literatur kann entschieden werden, ob die Maßnahme vor Ort sinnvoll ist. 


\section{Empfehlung}

Wie bisher kann von den Vorgaben der Richtlinie grundsätzlich dann abgewichen werden, wenn nach Prüfung alternativer Maßnahmen diese nicht zu ein em niedrigeren Schutzniveau für Patient und medizinischem Personal führen. Die entsprechenden Maßnahmen müssen im Fall der Abweichung von der Richtlinie fachlich begründet werden.

\section{Mitglieder der Arbeitsgruppe}

Martin Exner, Petra Ga stmeier, HeinzMichael Just, Axel Kramer, Joachim Martius, Martin Mielke, Alfred Nassauer, Roland Schulze-Röbbecke, Constanze Wendt (Leitung der Arbeitsgruppe)

\section{Literatur}

1. Exner M, Kistemann T, Unger G et al (1999) Zukünftige Präventions- und Kontrollstrategien in der Krankenhaushygiene. Bundesgesundheitsblatt 42:798-801

2. Kommission für Krankenhaushygiene und Infektionsprävention (2002) Anforderungen an die Hygiene bei der Aufbereitung flexibler Endoskope und endoskopischen Zusatzinstrumentariums. Bundesgesundheitsblatt 45:395-411

3. Kommission für Krankenhaushygiene und Infektionsprävention (2001) Anforderungen an die Hygiene bei der Aufbereitung von Medizinprodukten. Bundesgesundheitsblatt 44:1115-1126

4. Kommission für Krankenhaushygiene und Infektionsprävention (2004) Anforderungen an die Hygiene bei der Reinigung und Desinfektion von Flächen. Bundesgesundheitsblatt 47:51-61

5. Kommission für Krankenhaushygiene und Infektionsprävention (2002) Anforderungen der Hygiene an die baulich-funktionelle Gestaltung und apparative Ausstattung von Endoskopieeinheiten. Bundesgesundheitsblatt 45:412-414

6. Kommission für Krankenhaushygiene und Infektionsprävention (2000) Anforderungen der Hygiene bei Operationen und anderen invasiven Eingriffen. Bundesgesundheitsblatt 43:644-648

7. Kommission für Krankenhaushygiene und Infektionsprävention (2007) Empfehlung zur Prävention nosokomialer Infektionen bei neonatologischen Intensivpflegepatienten mit einem Geburtsgewicht unter $1500 \mathrm{~g}$. Bundesgesundheitsblatt 50:1265-1303

8. Kommission für Krankenhaushygiene und Infektionsprävention (2000) Empfehlungen zur Händehygiene. Bundesgesundheitsblatt 43:230-233

9. Kommission für Krankenhaushygiene und Infektionsprävention (2000) Empfehlungen zur Prävention der nosokomialen Pneumonie. Bundesgesundheitsblatt 43:302-309

10. Kommission für Krankenhaushygiene und Infektionsprävention (2007) Empfehlungen zur Prävention postoperativer Infektionen im Operationsgebiet. Bundesgesundheitsblatt 50:377-393

11. Kommission für Krankenhaushygiene und Infektionsprävention (1999) Empfehlungen zur Prävention und Kontrolle Katheter-assoziierter Harnwegsinfektionen. Bundesgesundheitsblatt 42:806809
12. Kommission für Krankenhaushygiene und Infektionsprävention (1999) Empfehlungen zur Prävention und Kontrolle von Methicillin-resistenten Staphylococcusaureus-Stämmen (MRSA) in Krankenhäusern und anderen medizinischen Einrichtungen. Bundesgesundheitsblatt 42:954-958

13. Kommission für Krankenhaushygiene und Infektionsprävention (2006) Infektionsprävention in der Zahnheilkunde - Anforderungen an die Hygiene. Bundesgesundheitsblatt 49:375-394

14. Kommission für Krankenhaushygiene und Infektionsprävention (2005) Infektionsprävention in Heimen. Bundesgesundheitsblatt 48:1061-1080

15. Kommission für Krankenhaushygiene und Infektionsprävention (2002) Prävention Gefäßkatheterassoziierter Infektionen. Bundesgesundheitsblatt 45:907-924 